\title{
Importância nutricional da vitamina D na prevenção da COVID-19: uma revisão
}

\section{sistemática}

\author{
Nutritional importance of vitamin D in the prevention of COVID-19: a systematic review \\ Importancia nutricional de la vitamina D en la prevención de COVID-19: una revisión sistemática
}

Recebido: 25/10/2021 | Revisado: 06/11/2021 | Aceito: 12/11/2021 | Publicado: 21/11/2021

Jerônimo Gregório da Silva Neto ORCID: https://orcid.org/0000-0003-1067-2604 Faculdade Estácio Teresina, Brasil

E-mail: jeronimogregg985@gmail.com

Byanka Pereira da Silva e Silva ORCID: https://orcid.org/0000-0001-5582-6422 Faculdade Estácio Teresina, Brasil E-mail: byankanutri1@gmail.com

Lucas Eduardo Alves de Oliveira ORCID: https://orcid.org/0000-0001-7125-3441 Faculdade Estácio Teresina, Brasil

E-mail: lucaseduardo66610@gamil.com

Amanda Cristine Ferreira dos Santos ORCID: https://orcid.org/0000-0002-1979-6745 Faculdade Estácio Teresina, Brasil E-mail: amanda.cristine@estacio.br

Carlos Henrique Ribeiro Lima ORCID: https://orcid.org/0000-0003-2947-7956 Faculdade Estácio Teresina, Brasil E-mail: lima.carlos@estacio.br

Lilia Maria Monteiro de Oliveira e Silva ORCID: https://orcid.org/0000-0001-7372-9335 Faculdade Estácio Teresina, Brasil E-mail: oliveira.lilia@estacio.br

Andrea Nunes Mendes de Brito ORCID: https://orcid.org/0000-0002-8270-9018 Faculdade Estácio Teresina, Brasil E-mail: andrea.brito@estacio.br

\begin{abstract}
Resumo
Objetivo: Verificar a importância nutricional da vitamina D na prevenção da Covid-19. Metodologia: Realizou-se uma revisão sistemática que seguiu os critérios do PRISMA - Principais Itens para Relatar Revisões Sistemáticas e Metaanálise. A busca dos artigos foi realizada nas bases de dados PubMed, Scielo, Lilacs e Biblioteca Virtual em Saúde, por meio dos descritores nos idiomas português e inglês: COVID-19", "Prevenção" e "Vitamina D". O acesso às bases de dados foi realizado no período de fevereiro à março de 2021. Resultados: Foram encontrados 250 artigos e após a aplicação dos critérios de inclusão restaram 13 artigos. Percebeu-se que a vitamina D desempenha um importante papel para diminuir os riscos e a severidade da COVID-19. A possível explicação do mecanismo de ação desta vitamina em doenças infecciosas como COVID-19 é por seu papel regulador na imunidade adquirida e na imunidade inata. Conclusão: A vitamina D pode ter um papel na prevenção da COVID-19, particularmente para pessoas com níveis abaixo do recomendado. Ela está ligada a ações anti-inflamatória, antiviral, antitrombótica, antioxidante e moduladora do sistema imune como sendo de grande valia ao diminuir o risco de infecção e a progressão da doença.
\end{abstract}

Palavras-chave: COVID-19; Prevenção; Vitamina D.

\section{Abstract}

Objective: To verify the nutritional importance of vitamin D in the prevention of Covid-19. Methodology: A systematic review was carried out which followed the PRISMA criteria - Main Items for Reporting Systematic Reviews and Metaanalysis. The search for articles was performed in the PubMed, Scielo, Lilacs and Virtual Health Library databases, using the descriptors in Portuguese and English: COVID-19", "Prevention" and "Vitamin D". Access to the databases was carried out from February to March 2021. Results: 250 articles were found and after applying the inclusion criteria, 13 articles remained. Vitamin D was found to play an important role in decreasing the risks and severity of COVID-19. The possible explanation for the mechanism of action of this vitamin in infectious diseases such as COVID-19 is its 
regulatory role in acquired immunity and innate immunity. Conclusion: Vitamin D may play a role in preventing COVID-19, particularly for people with levels below recommended levels. It is linked to anti-inflammatory, antiviral, antithrombotic, antioxidant and immune system modulating actions as being of great value in decreasing the risk of infection and disease progression.

Keywords: COVID-19; Prevention; D vitamin.

\section{Resumen}

Objetivo: Verificar la importancia nutricional de la vitamina D en la prevención de Covid-19. Metodología: Se realizó una revisión sistemática que siguió los criterios PRISMA - Principales ítems para reportar revisiones sistemáticas y metaanálisis. La búsqueda de artículos se realizó en las bases de datos PubMed, Scielo, Lilacs y Biblioteca Virtual en Salud, utilizando los descriptores en portugués e inglés: COVID-19 "," Prevención "y" Vitamina D ". El acceso a las bases de datos se realizó de febrero a marzo de 2021. Resultados: se encontraron 250 artículos y luego de aplicar los criterios de inclusión quedaron 13 artículos. Se encontró que la vitamina D juega un papel importante en la disminución de los riesgos y la gravedad del COVID-19. La posible explicación del mecanismo de acción de esta vitamina en enfermedades infecciosas como COVID-19 es su papel regulador en la inmunidad adquirida y la inmunidad innata. Conclusión: La vitamina D puede desempeñar un papel en la prevención de COVID-19, particularmente para personas con niveles por debajo de los niveles recomendados. Está relacionado con acciones antiinflamatorias, antivirales, antitrombóticas, antioxidantes y moduladoras del sistema inmunológico por ser de gran valor para disminuir el riesgo de infección y progresión de la enfermedad.

Palabras clave: COVID-19; Prevención; Vitamina D.

\section{Introdução}

O novo coronavírus-2019, denominada (COVID-19), é uma doença respiratória causada pelo vírus SARS-CoV-2. Em dezembro de 2019, a Organização Mundial de Saúde (OMS) começou a verificar um aumento de casos de pneumonia de causa desconhecida na cidade de Wuhan, na China. Em 7 de janeiro de 2020, as autoridades chinesas informaram que a causa era esse novo tipo de vírus. A OMS declarou, em 30 de janeiro de 2020, que o surto da doença pelo novo vírus constitui uma emergência de saúde pública de importância internacional (Santos, 2020), caracterizando-a, em 11 de março de 2020, como uma pandemia, desde então, todos os países vêm monitorando a progressão, o comportamento e as respostas dadas à essa doença.

O aspecto clínico da infecção é muito amplo, podendo variar de sintomas leves, como um simples resfriado, até mais graves como uma pneumonia severa e óbito. Os sintomas da COVID-19 começam a aparecer após o período de incubação, entre 02 e 14 dias após a infecção e a média de indivíduos que foram a óbito devido a complicações varia de 6 a 41 dias, o que dependerá da idade e do sistema imunológico de cada paciente (Filho, et al., 2020).

Nos últimos meses, várias estratégias de prevenção e tratamento foram implementadas, algumas baseadas na ciência, outras, em conhecimentos adquiridos por meio da observação. Nesse sentido, muitas evidências vêm surgindo para auxiliar no combate ao novo vírus, principalmente pessoas consideradas de risco, tais como: os portadores de doenças crônicas, idosos, gestantes, puérperas e crianças menores de 5 anos (Santos, 2020).

Apesar de ainda não existirem evidências suficientes, alguns estudos clínicos apoiam a suplementação de doses da Vitamina D em pessoas e/ou pacientes expostos ao SARS-CoV-2 como método de prevenção e diminuição dos sintomas, a verificação da hipótese parece estar cada vez mais próxima (Filho, et al., 2020).

Desde o século XX, a Vitamina D é conhecida devido seus diversos efeitos positivos no metabolismo humano sendo necessária para o bom funcionamento do organismo. A vitamina D pode ser um coadjuvante para o tratamento da COVID-19, desse modo à exposição ao sol juntamente com alimentação são essenciais para a produção. Alguns fatores são determinantes para a deficiência da vitamina como o uso excessivo de filtro solar e a restrição generalizada de idosos à exposição solar. (Kratz, et al., 2018).

A vitamina D e seus metabólitos são promissores para gerar respostas imunes inatas e adaptativas no organismo, sendo uma mediadora dos processos pró-inflamatórios e pós-inflamatórios. Assim, durante a atual pandemia, estudos mostraram que 
seus metabólitos são promissores para gerar respostas imunes inatas e adaptativas no organismo, sendo uma mediadora dos processos pró-inflamatórios e pós- inflamatórios de vírus como da gripe e outros que causam infecções. (Grant, et al., 2020).

Nessa lógica, Moreira (2018), fez um estudo em que a suplementação com vitamina D se mostrou protetiva contra infecções agudas do trato respiratório. Quando divididas em subgrupos, o subgrupo com níveis séricos $<25 \mathrm{nmol} / \mathrm{L}$ mostrou se beneficiar das suplementações $(P=0,002)$, porém o subgrupo com níveis de vitamina $\mathrm{D}>25 \mathrm{nmol} / \mathrm{L}$ não mostraram diferença estatisticamente significativa $(\mathrm{P}=0,15)$. A suplementação de vitamina $\mathrm{D}$, portanto, se mostrou segura e com raros efeitos adversos, mantendo os níveis dentro dos valores de referência, (hipercalcemia $0,5 \%$ e nefrolitíase $0,2 \%$ ).

Dados preliminares coletados atualmente em Torino indicam que os pacientes com Covid-19 têm uma alta prevalência de deficiência de vitamina D. Esta deficiência de vitamina generalizada pode ser compensada com a exposição ao sol, alimentos ricos em vitamina D e preparações farmacêuticas específicas sob supervisão médica ou de nutricionistas (Dultra, et al., 2020).

Diante do exposto, identifica-se a importância em ter uma visão abrangente sobre a vitamina $\mathrm{D}$ na prevenção da COVID-19, contemplando desde a captação dos profissionais da saúde até o desenvolvimento de protocolos clínicos como estratégia para a prevenção da doença e possíveis complicações futuras, além de auxiliar no planejamento local das ações de saúde pública. Assim, o estudo poderá servir como instrumento de apoio para tomada de decisões, pelos profissionais e famílias em caso de inconformidades.

Dessa maneira, o objetivo do presente estudo é realizar uma revisão sistemática de literatura sobre a importância nutricional da vitamina D na prevenção da Covid-19.

\section{Metodologia}

Trata-se de uma revisão sistemática norteada pelo questionamento: "Qual a importância da vitamina D para a prevenção da COVID-19?”. A revisão foi realizada seguindo as recomendações PRISMA (Principais itens para relatar Revisões sistemáticas e Meta-análises: A recomendação PRISMA", 2015, p. 335).

Realizou-se busca para seleção dos estudos em três bases de dados: Scientific Electronic Library Online-(SciELO), Literatura Latino-Americana em Ciências da Saúde (LILACS) e PubMed, e uma biblioteca virtual: Biblioteca Virtual em Saúde (BVS), usando os descritores de busca: "COVID-19", "Prevenção" e "Vitamina D" "Coronavirus infections" and "Prevention" and "Vitamin d".

Os critérios de elegibilidade foram: artigos originais (estudos do tipo ensaio clínico randomizado, estudo piloto, duplocego, retrospectivo, observacionais e testes controlados), relacionados ao tema de interesse desse estudo e publicados nos últimos dois anos (2020-2021) nos idiomas português e inglês. Excluiu-se artigos de revisão, resenhas, artigos duplicados e também aqueles com acesso indisponível nas plataformas digitais gratuitamente.

Para elaboração da revisão integrativa avaliou-se inicialmente os títulos, seguido da leitura dos resumos e posteriormente a leitura na íntegra dos estudos. O procedimento foi feito por três pesquisadores simultaneamente e de forma independente, levando em consideração os critérios de inclusão e exclusão pré-definidos. Os trabalhos para os quais houve discordância foram analisados em reunião com os autores para avaliação e consenso sobre a inclusão na revisão.

A extração dos dados foi realizada por meio de um protocolo elaborado pelos pesquisadores, no qual foram incluídos os seguintes dados: autor, ano, título, objetivo, método e resultados. A qualidade dos estudos foi avaliada por meio da ferramenta "Quality Assessment Tool For Quantitative Studies". Com base em cinco itens desta escala: 1) viés de seleção; 2) desenho de estudo; 3) fatores de confundimento; 4) métodos de coleta de dados; e 5) tipo de análise empregada para o desfecho, os estudos foram classificados em "fortes", "moderados" ou "fracos". Ao final, cada estudo recebeu uma pontuação da escala QATQS, 
sendo considerados fortes no caso de nenhum dos quesitos terem sido avaliados como fraco; moderado, no caso dos estudos que apresentaram um dos quesitos classificado como fraco; e, fracos, os estudos com um ou mais quesitos assim avaliados.

Por meio da investigação nas bases de dados foram encontrados 250 artigos, desses foram selecionados 53 dos últimos 2 anos, dos quais 21 eram duplicados e 19 não atendiam aos critérios de inclusão estabelecidos à priori. Resultaram, assim, 13 artigos (Figura 1).

Figura 1 - Fluxograma da seleção dos estudos nas bases de dados.

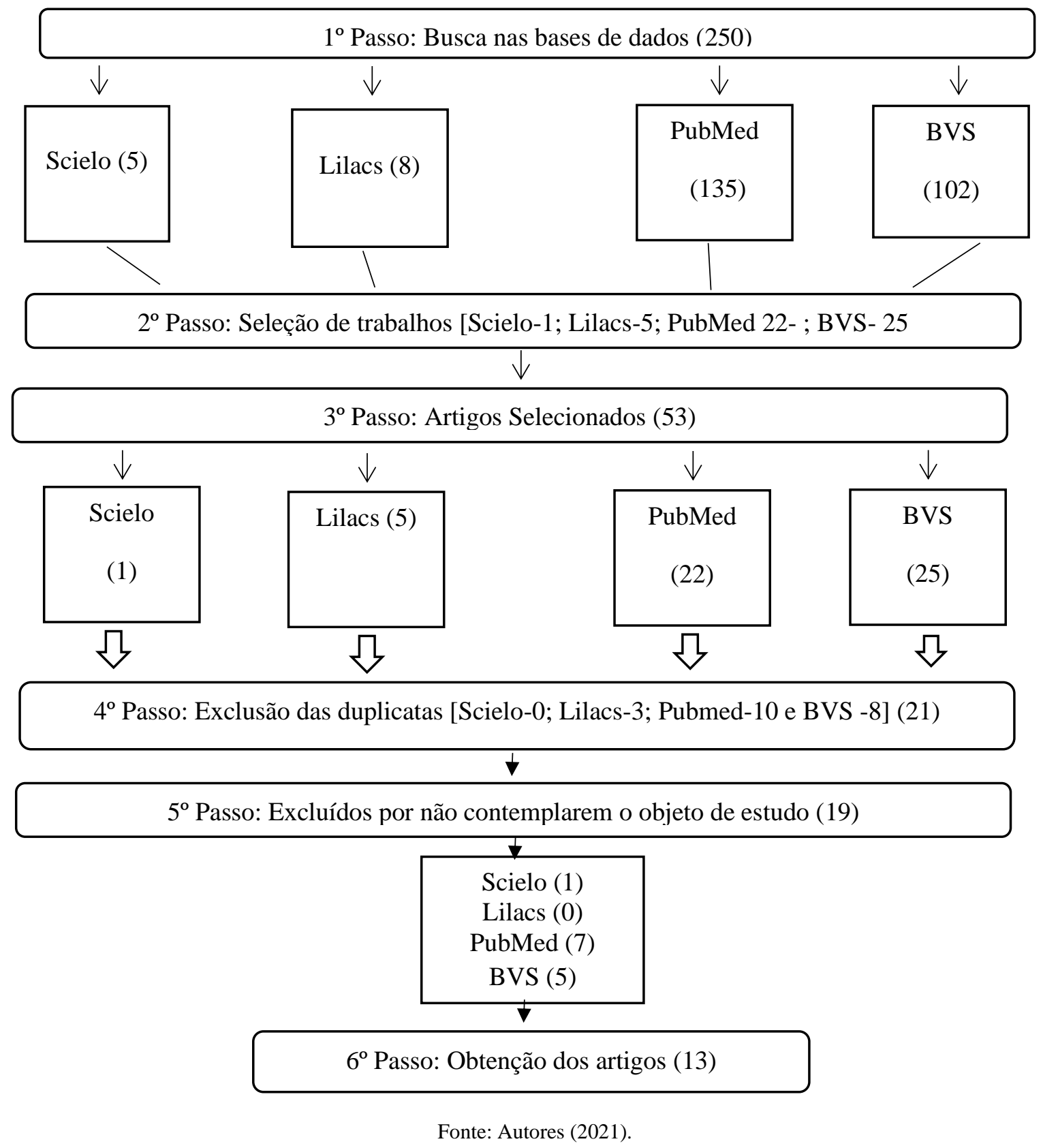

\section{Resultados e Discussão}

Na Tabela 1 pode-se observar a distribuição dos artigos, conforme o autor, ano, título, objetivo, tipo de estudo e resultados. 
Tabela 1- Distribuição das produções científicas sobre a interação da Vitamina D e Covid-19 publicadas no período de 2020 e 2021 segundo o autor, ano, objetivo, método e resultados.

\begin{tabular}{|c|c|c|c|}
\hline Autor/ano & Objetivo & Tipo de Estudo & Resultados \\
\hline $\begin{array}{l}\text { Murai et al, } \\
\quad 2021\end{array}$ & $\begin{array}{l}\text { Investigar o efeito } \\
\text { da administração de } \\
\text { vitamina } \mathrm{D}_{3} \text { no tempo } \\
\text { de internação hospitalar } \\
\text { e outros desfechos } \\
\text { clínicos relevantes e } \\
\text { eventos adversos em } \\
\text { pacientes hospitalizados } \\
\text { com COVID-19 } \\
\text { moderado a grave }\end{array}$ & $\begin{array}{l}\text { Estudo multicêntrico, duplo- } \\
\text { cego, de grupo paralelo, } \\
\text { randomizado e controlado por } \\
\text { placebo com } 1.240 \\
\text { pacientes avaliados. }\end{array}$ & 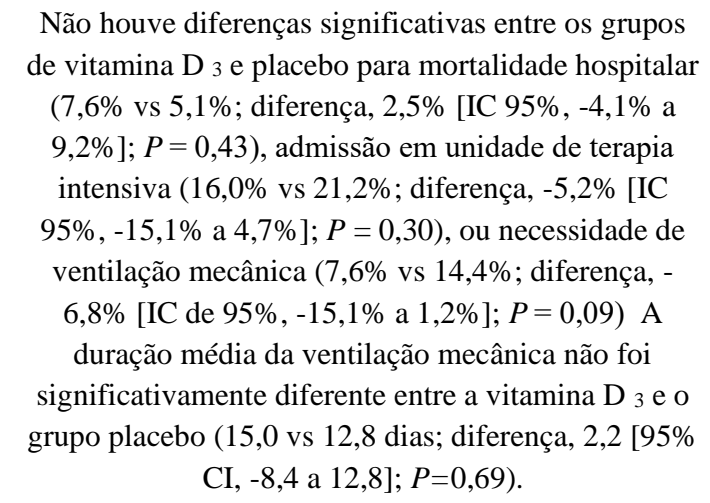 \\
\hline $\begin{array}{c}\text { Macaya et.al, } \\
2021\end{array}$ & $\begin{array}{l}\text { Explorar a associação } \\
\text { entre deficiência da } \\
\text { vitamina } \mathrm{D} \text { e o } \\
\text { desenvolvimento de } \\
\text { COVID-19 grave. }\end{array}$ & $\begin{array}{c}\text { Um estudo retrospectivo } \\
\text { realizado em uma coorte de } \\
\text { pacientes ( } 80 \text { pacientes com } \\
\text { média de } 67 \text { anos) atendidos no } \\
\text { departamento de emergência de } \\
\text { um hospital terciário em } \\
\text { Madrid, Espanha. }\end{array}$ & $\begin{array}{c}\text { Dos } 80 \text { pacientes, } 31 \text { (39\%) apresentaram que a } \\
\text { deficiência de vitamina D tendeu a predizer um risco } \\
\text { aumentado de desenvolver COVID-19 grave após } \\
\text { ajuste para idade, sexo, obesidade, doença cardíaca e } \\
\text { doença renal A idade teve uma interação negativa com } \\
\text { o efeito da deficiência no desfecho composto, } \\
\text { indicando que o efeito foi mais perceptível em idades } \\
\text { mais jovens. Além disso, o gênero masculino foi } \\
\text { associado com deficiência e com COVID-19 grave em } \\
\text { idades mais jovens. }\end{array}$ \\
\hline $\begin{array}{l}\text { Castillo et.al, } \\
2020\end{array}$ & $\begin{array}{l}\text { Avaliar o efeito do } \\
\text { tratamento com } \\
\text { calcifediol na admissão } \\
\text { em unidade de terapia } \\
\text { intensiva e na taxa de } \\
\text { mortalidade entre } \\
\text { pacientes espanhóis } \\
\text { hospitalizados por } \\
\text { COVID-19 }\end{array}$ & $\begin{array}{c}\text { Ensaio clínico duplo- } \\
\text { mascarado, piloto paralelo, } \\
\text { aleatório, de rótulo aberto com } \\
76 \text { pacientes consecutivos } \\
\text { hospitalizados com infecção por } \\
\text { COVID-19 e quadro clínico de } \\
\text { infecção respiratória aguda. }\end{array}$ & $\begin{array}{l}\text { De } 50 \text { pacientes tratados com calcifediol, um } \\
\text { necessitou de admissão na UTI ( } 2 \% \text { ), enquanto de } 26 \\
\text { pacientes não tratados, } 13 \text { precisou de admissão } \\
(50 \%) \text { valor de } \mathrm{p} \mathrm{X}^{2} \text { testes de Fischer } \mathrm{p} \\
<0,001 . \text { Razão de chances da estimativa de risco } \\
\text { univariada para UTI em pacientes com tratamento } \\
\text { com Calcifediol versus sem tratamento com } \\
\text { Calcifediol: } 0,02 \text { (IC } 95 \% \text { 0,002-0,17). Odds Ratio } \\
\text { de estimativa de risco multivariado para UTI em } \\
\text { pacientes com tratamento com calcifediol vs UTI sem } \\
\text { tratamento com calcifediol (ajuste por hipertensão e } \\
\text { DM2): 0,03 (IC 95\%: 0,003-0,25). Dos pacientes } \\
\text { tratados com calcifediol, nenhum morreu e todos } \\
\text { receberam alta hospitalar, sem complicações. Os } 13 \\
\text { pacientes não tratados com calcifediol, que não foram } \\
\text { admitidos na UTI, tiveram alta. Dos } 13 \text { pacientes } \\
\text { admitidos na UTI, dois morreram e os } 11 \text { restantes } \\
\text { tiveram alta. }\end{array}$ \\
\hline
\end{tabular}




\begin{tabular}{|c|c|c|c|}
\hline Ling et. al, 2020 & $\begin{array}{l}\text { Determinar se os níveis } \\
\text { séricos de } 25(\mathrm{OH}) \mathrm{D} \text { e / } \\
\text { ou a deficiência de } \\
\text { vitamina } \mathrm{D} \text { afetam a } \\
\text { mortalidade na infecção } \\
\text { por COVID-19 }\end{array}$ & $\begin{array}{c}\text { Estudo observacional } \\
\text { transversal multicêntrico } \\
\text { retrospectivo com um total de } \\
444 \text { participantes. A idade } \\
\text { mediana dos participantes era } \\
74 \text { (intervalo interquartil, IQR, } \\
63,83 \text { ) e } 199 \text { participantes eram } \\
\text { do sexo feminino }(44,9 \%) \text {. }\end{array}$ & $\begin{array}{l}\text { No coorte primário de } 444 \text { pacientes, a terapia de } \\
\text { reforço com colecalciferol foi associada a um risco } \\
\text { reduzido de mortalidade por COVID-19, após ajuste } \\
\text { para potenciais fatores de confusão. Este achado foi } \\
\text { replicado em uma coorte de validação de } 541 \\
\text { pacientes. O tratamento com terapia de reforço com } \\
\text { colecalciferol, independentemente dos níveis séricos } \\
\text { de } 25(\mathrm{OH}) \mathrm{D} \text { basais, parece estar associado a um } \\
\text { risco reduzido de mortalidade em pacientes } \\
\text { internados agudos internados com COVID-19. }\end{array}$ \\
\hline Sulli et.al, 2021 & $\begin{array}{c}\text { Correlacionar as } \\
\text { concentrações séricas de } \\
\text { 25OH-vitamina D com } \\
\text { parâmetros clínicos de } \\
\text { envolvimento pulmonar, } \\
\text { em pacientes idosos } \\
\text { hospitalizados por } \\
\text { infecção por SARS-CoV- } \\
2 .\end{array}$ & $\begin{array}{c}\text { Estudo de caso controle } \\
\text { com sessenta e cinco pacientes } \\
\text { consecutivos com COVID-19 } \\
\text { (idade média de } 76 \pm 13 \text { anos) e } \\
\text { sessenta e cinco indivíduos } \\
\text { controle pareados por sexo e } \\
\text { idade (CNT) foram } \\
\text { analisados. Os seguintes } \\
\text { parâmetros clínicos, incluindo } \\
\text { comorbidades, foram coletados } \\
\text { na admissão: tipo de } \\
\text { envolvimento pulmonar, } \\
\text { parâmetros respiratórios (PaO } 2, \\
\text { SO 2, PaCO 2, PaO } 2 \text { / FiO 2), } \\
\text { parâmetros laboratoriais } \\
\text { (incluindo 25OH-vitamina } \mathrm{D}_{\text {, }} \\
\text { D-dímero, proteína C reativa). }\end{array}$ & 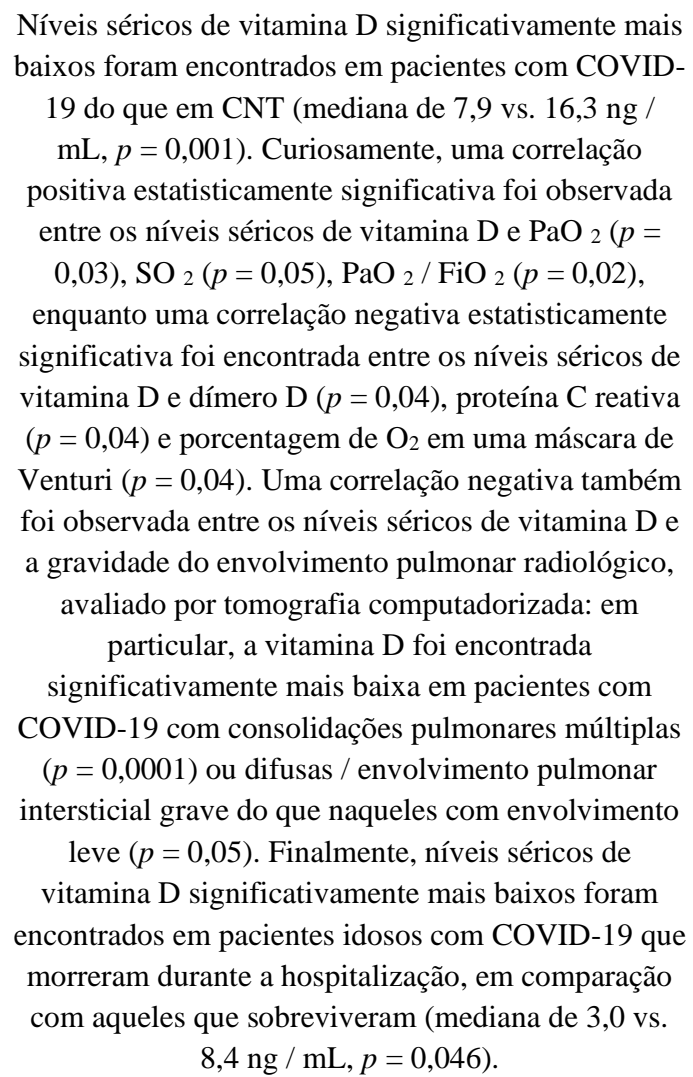 \\
\hline
\end{tabular}




\begin{tabular}{|c|c|c|c|}
\hline $\begin{array}{l}\text { Ricci, et.al, } \\
\quad 2021\end{array}$ & $\begin{array}{l}\text { Avaliar se a deficiência de } \\
\text { Vit } D \text { foi um fator de } \\
\text { risco para desenvolver } \\
\text { quadros clínicos mais } \\
\text { graves e } \\
\text { comprometimento } \\
\text { pulmonar mais grave em } \\
\text { pacientes com infecção } \\
\text { por coronavírus. }\end{array}$ & $\begin{array}{l}\text { Ensaio clínico. } 52 \text { pacientes } \\
\text { hospitalizados afetados com } \\
\text { infecção por Covid-19 e com } \\
\text { diferentes graus de } \\
\text { envolvimento pulmonar. }\end{array}$ & $\begin{array}{l}\text { Os níveis de vitamina D eram deficientes em }(80 \%) \text { dos } \\
\text { pacientes, insuficientes em }(6,5 \%) \text { e normais } \\
(13,5 \%) \text {. Pacientes com níveis plasmáticos de vitamina } \\
\text { D muito baixos tinham valores de dímero D mais } \\
\text { elevados, contagem de linfócitos B mais elevada, } \\
\text { redução de linfócitos T CD8 + com razão CD4 / CD8 } \\
\text { baixa, achados clínicos mais comprometidos (medidos } \\
\text { por escores LIPI e SOFA) e envolvimento de } \\
\text { tomografia computadorizada de tórax. }\end{array}$ \\
\hline $\begin{array}{c}\text { Annweiler et.al, } \\
2020\end{array}$ & $\begin{array}{c}\text { Determinar se a } \\
\text { suplementação de } \\
\text { vitamina D tomada } \\
\text { regularmente durante o } \\
\text { ano anterior ou após o } \\
\text { diagnóstico de COVID-19 } \\
\text { foi eficaz na melhoria da } \\
\text { sobrevida entre pacientes } \\
\text { idosos frágeis com } \\
\text { COVID-19 }\end{array}$ & $\begin{array}{l}\text { Estudo quase experimental } \\
\text { conduzido em uma unidade } \\
\text { geriátrica de cuidados agudos } \\
\text { dedicada a pacientes com } \\
\text { COVID-19. Os dados do estudo } \\
\text { foram coletados } \\
\text { retrospectivamente dos } \\
\text { prontuários de } 77 \text { pacientes. Os } \\
\text { grupos de intervenção foram } \\
\text { participantes regularmente } \\
\text { suplementados com vitamina D } \\
\text { durante o ano anterior (Grupo } \\
\text { 1), e aqueles suplementados } \\
\text { com vitamina D após o } \\
\text { diagnóstico de COVID-19 } \\
\text { (Grupo 2). O grupo comparador } \\
\text { envolveu participantes que não } \\
\text { receberam suplementos de } \\
\text { vitamina D (Grupo 3). }\end{array}$ & $\begin{array}{l}\text { Os três grupos ( } n=77 \text {; média } \pm \text { DP, } 88 \pm 5 \text { anos; } 49 \% \\
\text { mulheres) foram semelhantes no início do estudo } \\
\text { (exceto para a proporção de mulheres, } p=0,02) \text {, assim } \\
\text { como os tratamentos usados para COVID-19. No } \\
\text { Grupo } 1 \text { ( } n=29), 93,1 \% \text { dos participantes do COVID- } \\
19 \text { sobreviveram no dia 14, em comparação com } 81,2 \% \\
\text { dos sobreviventes do Grupo } 2(n=16)(p=0,33) \text { e } \\
68,7 \% \text { dos sobreviventes do Grupo } 3(n=32)(p= \\
0,02 \text { ). Considerando o Grupo } 3 \text { como referência (razão } \\
\text { de risco }(\mathrm{HR})=1 \text { ), o HR totalmente ajustado para } \\
\text { mortalidade em } 14 \text { dias foi HR }=0,07 \text { ( } p=0,017 \text { ) para } \\
\text { o Grupo } 1 \text { e HR }=0,37 \text { ( } p=0,28 \text { ) para o Grupo } 2 \text {. O } \\
\text { Grupo } 1 \text { teve maior tempo de sobrevivência do que o } \\
\text { Grupo } 3 \text { (log-rank } p=0,015 \text { ), embora não tenha havido } \\
\text { diferença entre os Grupos } 2 \text { e } 3 \text { (log-rank } p=0,32 \text { ). O } \\
\text { Grupo } 1 \text {, mas não o Grupo } 2(p=0,40) \text {, foi associado a } \\
\text { menor risco de escore OSCI } \geq 5 \text { em comparação ao } \\
\text { Grupo } 3 \text { (odds ratio }=0,08, p=0,03 \text { ). }\end{array}$ \\
\hline $\begin{array}{c}\text { Pizzini et.al, } \\
2020\end{array}$ & $\begin{array}{c}\text { Investigar associações do } \\
\text { status de VITD à } \\
\text { apresentação da doença } \\
\text { no registro CovILD. }\end{array}$ & $\begin{array}{c}\text { Estudo prospectivo, } \\
\text { multicêntrico e observacional, } \\
\text { estudo incluiu } 22 \text { pacientes } \\
\text { externos e } 87 \text { hospitalizados, } \\
\text { dos quais } 18 \text { pacientes } \\
\text { necessitaram de tratamento na } \\
\text { UTI. }\end{array}$ & $\begin{array}{l}\text { Um total de } 109 \text { pacientes foram incluídos na análise } \\
\text { (60\% homens, } 40 \% \text { mulheres), com idade de } 58 \pm 14 \\
\text { anos. Oito semanas após o início de COVID-19, uma } \\
\text { alta proporção de pacientes apresentou } \\
\text { comprometimento do metabolismo de VITD e níveis } \\
\text { elevados de hormônio da paratireóide (PTH). As } \\
\text { concentrações de PTH aumentaram em pacientes que } \\
\text { necessitaram de tratamento em unidade de terapia } \\
\text { intensiva (UTI), enquanto os níveis de VITD não foram } \\
\text { significativamente diferentes entre os grupos de } \\
\text { gravidade da doença. Níveis baixos de VITD no início } \\
\text { da doença ou no acompanhamento de oito semanas não } \\
\text { foram relacionados à carga persistente de sintomas, } \\
\text { comprometimento da função pulmonar, inflamação } \\
\text { contínua ou anormalidades mais graves na TC. }\end{array}$ \\
\hline
\end{tabular}




\begin{tabular}{|c|c|c|c|}
\hline Notz et al, 2021 & $\begin{array}{l}\text { Analisar os efeitos } \\
\text { clínicos e imunológicos } \\
\text { dos níveis de vitamina D } \\
\text { em pacientes que sofrem } \\
\text { de (COVID-19) induzida } \\
\text { pela síndrome do } \\
\text { desconforto respiratório } \\
\text { agudo (SDRA) }\end{array}$ & $\begin{array}{l}\text { Estudo retrospectivo em um } \\
\text { único centro em pacientes } \\
\text { recebendo tratamento intensivo } \\
\text { com infecção confirmada por } \\
\text { SARS-CoV-2 e ARDS por } \\
\text { COVID-19 }\end{array}$ & $\begin{array}{l}\text { Um total de } 39 \text { pacientes com ARDS COVID-19 eram } \\
\text { elegíveis, dos quais } 26 \text { foram incluídos neste estudo } \\
\text { porque os dados sobre o status da vitamina D estavam } \\
\text { disponíveis. } 96 \% \text { sofriam de ARDS COVID-19 } \\
\text { grave. Todos os pacientes sem suplementação anterior } \\
\text { de vitamina } \mathrm{D}(\mathrm{n}=22 \text { ) tinham níveis séricos deficientes } \\
\text { de } 25 \text {-hidroxivitamina } \mathrm{D} \text {. A suplementação de vitamina } \\
\mathrm{D} \text { resultou em níveis séricos mais elevados de } 25 \text { - } \\
\text { hidroxivitamina } \mathrm{D} \text {, mas não aumentou os níveis de } \\
1,25 \text {-diidroxivitamina } \mathrm{D} \text { após } 10-15 \text { dias. Os } \\
\text { parâmetros clínicos não diferiram entre os pacientes } \\
\text { com níveis suficientes ou deficientes de } 25 \text { - } \\
\text { hidroxivitamina } \mathrm{D} \text {. Apenas plasmablastos circulantes } \\
\text { foram maiores em pacientes com níveis de } 25 \text { - } \\
\text { hidroxivitamina } \mathrm{D} \geq 30 \mathrm{ng} / \mathrm{ml}(\mathrm{p}=0,029 \text { ). Pacientes } \\
\text { com níveis de } 1,25 \text {-diidroxivitamina } \mathrm{D} \text { abaixo de } 20 \mathrm{pg} \\
\text { / ml necessitaram de ventilação mecânica mais } \\
\text { prolongada ( } \mathrm{p}=0 \text {. }\end{array}$ \\
\hline $\begin{array}{l}\text { Rastogi et al, } \\
2020\end{array}$ & $\begin{array}{l}\text { Efeito da suplementação } \\
\text { oral de colecalciferol em } \\
\text { altas doses na eliminação } \\
\text { do vírus SARS-CoV-2. }\end{array}$ & $\begin{array}{l}\text { Estudo Randomizado, controlado } \\
\text { por placebo. }\end{array}$ & $\begin{array}{l}\text { Quarenta indivíduos com ARN positivo para SARS- } \\
\text { CoV-2 foram randomizados para A linha de base sérica } \\
25(\mathrm{OH}) \mathrm{D} \text { foi de } 8,6(7,1 \text { a } 13,1) \text { e } 9,54(8,1 \text { a } 12,5) \mathrm{ng} \\
\text { / ml (p = 0,730), no grupo intervenção e controle, } \\
\text { respectivamente. } 10 \text { de } 16 \text { pacientes poderiam atingir } \\
25(\mathrm{OH}) \mathrm{D}>50 \mathrm{ng} \text { / ml no dia } 7 \text { e outros dois no dia } 14 \\
\text { [dia } 1425(\mathrm{OH}) \mathrm{D} \text { níveis de } 51,7(48,9 \text { a } 59,5) \mathrm{ng} / \mathrm{ml} \text { e } \\
15,2(2,7 \text { a 19,5) ng / ml (p <0,001) no grupo } \\
\text { intervenção e controle, respectivamente]. Uma dose } \\
\text { alta de suplementação oral de vitamina D para } \\
\text { aumentar } 25 \text { (OH) D> } 50 \text { ng / ml ajudou a atingir a } \\
\text { negatividade do RNA de SARS-CoV-2 em maior } \\
\text { proporção de indivíduos com deficiência de vitamina D } \\
\text { assintomáticos com infecção por SARS-CoV-2 junto } \\
\text { com uma diminuição significativa no marcador } \\
\text { inflamatório. A negatividade do RNA do SARS-CoV-2 } \\
\text { pela suplementação com colecalciferol pode ajudar a } \\
\text { reduzir as taxas de transmissão da infecção altamente } \\
\text { contagiosa do SARS-CoV-2. Uma garantia para os } \\
\text { trabalhadores de saúde pública em relação à maior } \\
\text { probabilidade de SARS CoV-2 RNA negatividade em } \\
\text { indivíduos recebendo suplementação terapêutica de } \\
\text { colecalciferol será encorajadora. }\end{array}$ \\
\hline$\frac{\text { Abdollahi et al }}{2020}$ & $\begin{array}{l}\text { Explorar uma associação } \\
\text { entre o nível sérico de } \\
\text { vitamina } \mathrm{D} \text {, obesidade e } \\
\text { condições de saúde } \\
\text { subjacentes, bem como a } \\
\text { vulnerabilidade ao } \\
\text { COVID-19 na população } \\
\text { iraniana. }\end{array}$ & $\begin{array}{l}\text { Estudo de caso-controle de } 201 \\
\text { pacientes com infecção por } \\
\text { coronavírus e } 201 \\
\text { controles. Casos e controles } \\
\text { foram pareados por idade e } \\
\text { sexo. O estudo foi realizado por } 2 \\
\text { meses (fevereiro de } 2020 \text { a abril } \\
\text { de 2020) no Complexo Hospitalar } \\
\text { Imam Khomeini, Teerã, Irã. }\end{array}$ & $\begin{array}{l}\text { Uma correlação negativa significativa }(p=0,02) \text { foi } \\
\text { observada entre o nível sérico de vitamina D e o } \\
\text { desenvolvimento de infecção por coronavírus. Além } \\
\text { disso, os resultados mostraram que os casos COVID - } \\
19 \text { eram mais propensos a ter excesso de peso do que os } \\
\text { controles ( } p=0,023) \text {. Diabetes mellitus, hipertensão e } \\
\text { infecções respiratórias foram encontrados em } 20,89 \% \text {, } \\
9,65 \% \text { e } 6,96 \% \text { dos casos, respectivamente. }\end{array}$ \\
\hline $\begin{array}{c}\text { Maghbooli et, al } \\
2020\end{array}$ & $\begin{array}{l}\text { Investigar a associação } \\
\text { entre os níveis séricos de } \\
\text { 25-hidroxivitamina D e } \\
\text { seu efeito em resultados } \\
\text { clínicos adversos e }\end{array}$ & $\begin{array}{l}\text { Estudo transversal de um banco } \\
\text { de dados COVID-19 no hospital } \\
\text { Sina, Teerã, Irã. }\end{array}$ & $\begin{array}{l}\text { 74\% tinham infecção grave por COVID-19 e 32,8\% } \\
\text { tinham vitamina D suficiente. Após o ajuste para } \\
\text { fatores de confusão, houve uma associação significativa } \\
\text { entre a suficiência de vitamina D e a redução na } \\
\text { gravidade clínica, os níveis séricos de mortalidade de }\end{array}$ \\
\hline
\end{tabular}


Research, Society and Development, v. 10, n. 15, e102101522404, 2021

(CC BY 4.0) | ISSN 2525-3409 | DOI: http://dx.doi.org/10.33448/rsd-v10i15.22404

\begin{tabular}{|c|c|c|c|}
\hline & $\begin{array}{c}\text { parâmetros de função } \\
\text { imunológica e } \\
\text { mortalidade devido a uma } \\
\text { infecção por SARS-CoV- } \\
2 .\end{array}$ & & $\begin{array}{l}\text { pacientes internados de proteína C reativa (PCR) e um } \\
\text { aumento na porcentagem de linfócitos. Apenas } 9,7 \% \\
\text { dos pacientes com mais de } 40 \text { anos que tinham } \\
\text { vitamina D suficiente sucumbiram à infecção, em } \\
\text { comparação com } 20 \% \text { que tinham um nível circulante } \\
\text { de } 25(\mathrm{OH}) \mathrm{D}<30 \mathrm{ng} \text { / ml. A redução significativa na } \\
\text { PCR sérica, um marcador inflamatório, junto com o } \\
\text { aumento da porcentagem de linfócitos sugere que a } \\
\text { suficiência de vitamina D também pode ajudar a } \\
\text { modular a resposta imune, possivelmente reduzindo o } \\
\text { risco de tempestade de citocinas em resposta a essa } \\
\text { infecção viral. }\end{array}$ \\
\hline $\begin{array}{l}\text { Charoenngam } \\
\text { et, al } 2021\end{array}$ & $\begin{array}{l}\text { Determinar a associação } \\
\text { entre o status da vitamina } \\
\text { D e a morbidade e } \\
\text { mortalidade em pacientes } \\
\text { adultos hospitalizados } \\
\text { com doença por } \\
\text { coronavírus em 2019 } \\
\text { (COVID-19) }\end{array}$ & $\begin{array}{l}\text { Estudo retrospectivo de revisão } \\
\text { de prontuários em pacientes com } \\
\text { COVID-19 com idade } \geq 18 \text { anos } \\
\text { hospitalizados no Boston } \\
\text { University Medical Center entre } \\
1^{\circ} \text { de março e } 4 \text { de agosto de } \\
2020 .\end{array}$ & $\begin{array}{l}\text { Entre os } 287 \text { pacientes, } 100(36 \%) \text { tinham vitamina D } \\
\text { suficiente ( } 25[\mathrm{OH}] \mathrm{D}>30 \mathrm{ng} / \mathrm{mL}) \text { e } 41(14 \%) \\
\text { morreram durante a hospitalização. A análise } \\
\text { multivariada em pacientes com idade } \geq 65 \text { anos revelou } \\
\text { que a suficiência de vitamina } \mathrm{D}(25[\mathrm{OH}] \mathrm{D} \geq 30 \mathrm{ng} / \\
\text { mL) foi estatisticamente significativamente associada à } \\
\text { diminuição das chances de morte (OR ajustado } 0,33 \text {, IC } \\
\text { 95\%, } 0,12-0,94) \text {, respiratório agudo síndrome de } \\
\text { angústia (OR ajustado } 0,22 \text {, IC de } 95 \%, 0,05-0,96) \text { e } \\
\text { sepse grave / choque séptico (OR ajustado } 0,26, \mathrm{IC} \text { de } \\
\text { 95\%, 0,08-0,88), após ajuste para potenciais fatores de } \\
\text { confusão. }\end{array}$ \\
\hline
\end{tabular}

Fonte: Dados da pesquisa no PubMed, Scielo, Lilacs e BVS.

A partir das análises dos artigos percebeu-se que a vitamina D desempenha um importante papel para diminuir os riscos e a severidade da COVID-19. A possível explicação do mecanismo de ação desta vitamina em doenças infecciosas como COVID19 é por seu papel regulador na imunidade adquirida e na imunidade inata. Existe uma interação complexa entre a vitamina D, a infecção e o sistema imunológico. Para ajudar a regular a imunidade inata, 1,25 (OH) 2D é produzido em macrófagos em resposta à estimulação de receptores toll-like pela ligação de um agente infeccioso. Assim, a 1,25 (OH) D se liga ao VDR no macrófago, resultando em um aumento na produção de peptídeos antimicrobianos (AMPs), como defensina e catelicidina, que têm efeitos antivirais (Maghbooli, et al., 2020; (Charoenngam et al., 2021).

Castillo, et al., (2020) demonstraram que a administração de calcifediol pode melhorar o resultado clínico de indivíduos que necessitam de hospitalização para COVID-19. Ainda não se sabe se essa informação se aplica a pacientes em estágio inicial da doença e se o status basal de vitamina D modifica esses resultados.

Ling, et al., (2020) em seu estudo transversal buscaram determinar se os níveis séricos de 25 (OH) D e / ou a deficiência de vitamina D afetam a mortalidade na infecção por COVID-19 e verificaram que a terapia de reforço com colecalciferol em altas doses, independentemente dos níveis séricos basais de $25(\mathrm{OH}) \mathrm{D}$, está associada a um risco reduzido de mortalidade em pacientes internados com COVID-19.

Já Sulli, et al., (2021) observaram que a deficiência de vitamina D é um fator de risco para infecções, bem como para reatividade insuficiente do sistema imunológico inato, portanto, os status de vitamina D sérica (níveis mais baixos esperados) deve ser obrigatório como parte do estado de verificação de saúde regular.

No entanto, Murai, et al., (2021) em seu estudo randomizado e controlado por placebo que buscava investigar o efeito da administração de vitamina D no tempo de internação em pacientes hospitalizados com COVID-19, observou que uma única dose elevada de vitamina $\mathrm{D}$, em comparação com o placebo, não reduziu significativamente o tempo de internação ou melhorou 
quaisquer outros resultados clinicamente relevantes entre pacientes. Dessa maneira, os resultados não apoiam o uso de vitamina D para o tratamento de COVID-19 moderado a grave.

Porém, os resultados do estudo de Rastogi, et al., (2020) também randomizado viram que uma maior proporção de indivíduos com deficiência de vitamina D com infecção por SARS-CoV-2 tornaram o RNA SARS-CoV-2 negativo com uma diminuição significativa no fibrinogênio com a suplementação de colecalciferol em altas doses ao contrário da única dose elevada do estudo anterior.

No que diz respeito a COVID-19 grave e a síndrome do desconforto respiratório agudo (SDRA), Notz et al, (2021) observaram que existe deficiência de vitamina D nos pacientes. Porém, o status de 25-hidroxivitamina D não é relacionado a mudanças no curso clínico, enquanto baixos níveis de 1,25-di-hidroxivitamina D já são associados com ventilação mecânica prolongada e uma piora.

Ricci, et al., (2021) afirma que a deficiência de vitamina D está associada a respostas inflamatórias comprometidas e maior envolvimento pulmonar em pacientes afetados por COVID-19 grave. Porém, Pizzini, et al., (2020) demonstrou que as concentrações de $25(\mathrm{OH})$ D não se associam com inflamação persistente ou persistência dos sintomas. No entanto, os indivíduos com doença grave exibem um eixo paratireoide-vitamina D perturbado durante sua fase de recuperação, provavelmente devido à hospitalização prolongada. Embora a questão sobre causalidade ou consequência não possa ser respondida por meio dos dados, recomenda-se a realização de mais estudos intervencionistas para compreender corretamente o papel da vitamina D no COVID19, uma vez que o impacto dos efeitos da vitamina no manejo clínico de COVID-19 permanece indefinido.

Os estudos contemplados com o tema "Vitamina D, COVID-19 e Idade", indicaram que pacientes idosos frágeis hospitalizados com COVID-19 em que a suplementação regular de vitamina D3 foi associada a COVID-19 menos grave tem melhor taxa de sobrevida. A suplementação de vitamina D3 pode representar um tratamento adjuvante eficaz, acessível e bem tolerado para COVID-19 (Hammerschmidt \& Santana, 2020).

Nessa lógica, Seijo, e Oliveri, B, (2020) verificaram que idosos com deficiência de vitamina D no momento de testes da COVID-19 estão em maior risco de serem positivos para COVID-19 em comparação com aqueles indivíduos com status suficiente de vitamina D.

Enquanto Macaya, et al., (2021), em seu estudo retrospectivo, mostrou que a deficiência de vitamina D se associou com infecção COVID-19 grave. Essa associação foi modificada significativamente com a idade, indicando que a deficiência da vitamina pode ter um impacto maior no subgrupo de pacientes mais jovem. Além disso, o gênero masculino foi associado a deficiência de vitamina $\mathrm{D}$ e a resultados adversos em pacientes mais jovens. O impacto do gênero masculino nos desfechos não é totalmente compreendido, mas tem sido sugerido que os hormônios sexuais podem influenciar a expressão e / ou função da enzima conversora de angiotensina 2, e isso pode ser aumentado no cenário de deficiência de vitamina D.

Um outro ponto também importante tratado por Abdollahi, et al., (2020) foi a "Vitamina D, COVID-19 e Obesidade", observaram uma correlação negativa significativa entre o nível sérico de vitamina $\mathrm{D}$ e o desenvolvimento de infecção por coronavírus. Além disso, os resultados mostraram que os casos de COVID-19 eram mais propensos a apresentar excesso de peso do que os controles. Diabetes mellitus, hipertensão e infecções respiratórias foram encontrados em 20,89\%, 9,65\% e 6,96\% dos casos, respectivamente. Entretanto, essas condições de saúde subjacentes não foram significativamente diferentes entre casos e controles. Desse modo, a deficiência de vitamina $\mathrm{D}$ e a obesidade são os dois principais fatores predisponentes associados à vulnerabilidade à infecção por COVID-19.

Por fim, um aspecto identificado por Oliveira et al, (2020) é que os resultados obtidos não são unânimes ao definir a dose ideal de vitamina D para a suplementação de quais quer pacientes com COVID-19. Sobre isso, destacam que os profissionais de saúde precisam avaliar cada paciente tendo em vista a fisiopatologia da doença e os fatores que podem prejudicar o tratamento dos pacientes. 
A qualidade dos artigos, conforme o instrumento “Quality Assessment Tool For Quantitative Studies” está apresentada no Quadro 1.

Quadro 1 - Qualidade dos estudos analisados.

\begin{tabular}{|c|c|c|c|c|c|c|}
\hline Referência & Seleção de viés & $\begin{array}{l}\text { Delineamento } \\
\text { do estudo }\end{array}$ & Confundimento & $\begin{array}{c}\text { Métodos e } \\
\text { Coleta dos } \\
\text { dados }\end{array}$ & Perdas & $\begin{array}{c}\text { Classificação } \\
\text { Global }\end{array}$ \\
\hline $\begin{array}{l}\text { Murai et al, } \\
2021\end{array}$ & $\checkmark$ & $\checkmark$ & $\checkmark$ & + & + & $\checkmark$ \\
\hline $\begin{array}{c}\text { Macaya et.al, } \\
2021\end{array}$ & $\checkmark$ & + & + & $\checkmark$ & + & + \\
\hline $\begin{array}{l}\text { Castillo et.al, } \\
2020\end{array}$ & $\checkmark$ & + & $\checkmark$ & $\checkmark$ & $\checkmark$ & $\checkmark$ \\
\hline Ling et. al, 2020 & - & - & + & + & - & - \\
\hline Sulli et.al, 2021 & $\checkmark$ & + & $\checkmark$ & $V$ & $\checkmark$ & $\checkmark$ \\
\hline $\begin{array}{c}\text { Annweiler } \text { et.al, } \\
2020\end{array}$ & $\checkmark$ & + & $\checkmark$ & + & + & + \\
\hline $\begin{array}{l}\text { Ricci, et.al, } \\
\quad 2021\end{array}$ & $\checkmark$ & + & $\checkmark$ & $\checkmark$ & + & $\checkmark$ \\
\hline $\begin{array}{l}\text { Pizzini et.al, } \\
2020\end{array}$ & $\checkmark$ & + & + & + & $\checkmark$ & + \\
\hline Notz et al, 2021 & + & + & $\checkmark$ & $\checkmark$ & $\checkmark$ & $\checkmark$ \\
\hline $\begin{array}{l}\text { Rastogi et al, } \\
2020\end{array}$ & $\checkmark$ & $\checkmark$ & + & $\checkmark$ & + & $V$ \\
\hline$\frac{\text { Abdollahi }}{2020}$ et al, & + & + & + & $\checkmark$ & + & + \\
\hline $\begin{array}{l}\text { Maghbooli } e t, a l \\
2020\end{array}$ & + & - & + & - & - & - \\
\hline $\begin{array}{c}\text { Charoenngam } \\
e t, \text { al } 2021\end{array}$ & $\checkmark$ & + & $\checkmark$ & $V$ & $\checkmark$ & $\checkmark$ \\
\hline
\end{tabular}

- Fraco + Moderado $\checkmark$ Forte. Fonte: Autores.

Apesar dos resultados relevantes, esta revisão apresentou limitações. A variação metodológica dos estudos foi um fator limitante. Apesar da maioria dos artigos serem randomizados, os estudos transversais de Ling, et al., (2020) e Maghbooli, et al., (2020) que por design não podem ser determinados com certeza, demonstrando a importância de pesquisas futuras baseadas em estudos longitudinais para fornecer mais evidências, visto que a COVID- 19 é uma doença que ainda está sendo estudada e tem muito a se descobrir sobre ela e sua relação com a vitamina D. além disso, a principal limitação dos estudos foi referente a retirada e saídas (perdas), pois os pacientes apresentavam várias doenças e eram submetidos a medicamentos diversos que poderia afetar amostra ou incluíram um baixo número de pacientes.

\section{Conclusão}

Dado o exposto, constatou-se que a suplementação de vitamina D auxilia na prevenção da COVID-19 especialmente para indivíduos com baixos níveis do nutriente e melhora dos sintomas da doença (diminuição da incidência e o tempo de 
infecção, duração da internação hospitalar, admissão em Unidade de Tratamento intensivo, além da diminuição do período de incubação e da mortalidade). Desse modo, ela exerce ações anti-inflamatória, antiviral, antitrombótica, antioxidante, moduladora do sistema imune e diminui o risco de infecção e consequentemente a progressão da doença. Destaca-se que os resultados obtidos não são unânimes ao definir a dose ideal de vitamina D para a suplementação de pacientes com COVID-19.

Em função da quantidade de pesquisas finalizadas envolvendo o uso da vitamina D e COVID-19 até o momento, recomenda-se para trabalhos futuros a realização de ensaios clínicos randomizados e estudos multicêntricos que avaliem os efeitos dessas terapias em diferentes grupos e que estabeleçam se a carência de vitamina $\mathrm{D}$ se enquadra como fator de risco para agravamento da doença. Assim, será possível avaliar os efeitos da vitamina D na COVID-19 através de artigos com bons níveis de evidência.

\section{Referências}

Abdollahi, A., Sarvestani, HK., Rafat, Z., Ghaderkhani, S., Mahmoudi-Aliabadi, M., Jafarzadeh, B., Mehrtash, V. (2021). A associação entre o nível sérico de $25(\mathrm{OH})$ vitamina $\mathrm{D}$, obesidade e doenças subjacentes com o risco de desenvolver infecção por COVID-19: um estudo caso-controle de pacientes hospitalizados em Teerã, Irã. J Med Virol. 93:2359-2364. https://doi.org/10.1002/jmv.26726

Annweiler, G., Corvaisier, M., Gautier, J., Dubée, V., Legrand, E., Sacco, G., \& Annweiler, C. (2020). Vitamin D Supplementation Associated to Better Survival in Hospitalized Frail Elderly COVID-19 Patients: The GERIA-COVID Quasi-Experimental Study. Nutrients, 12(11), 3377. https://doi.org/10.3390/nu12113377

Araujo, F., Sawamura, M., Costa. (2020). Pneumonia por COVID-19: qual o papel da imagem no diagnóstico. J. bras. pneumol, v. 46, n.2, p. 144-150. https://doi.org/10.36416/1806-3756/e20200114

Castillo, E., Entrenas Costa, L. M., Vaquero, B., J. M., Alcalá Díaz, J. F., López Miranda, J., Bouillon, R., \& Quesada Gomez, J. M. (2020). “Efeito do tratamento com calcifediol e melhor terapia disponível versus melhor terapia disponível na admissão à unidade de terapia intensiva e mortalidade entre pacientes hospitalizados por COVID-19: um estudo clínico piloto randomizado". The Journal of steroid biohemistry and molecular biology, 203, 105751. https://doi.org/10.1016/j.jsbmb.2020.105751

Charoenngam, M.D., Arash, S., Reddy, N., Vodopivec, D., Apovian, C., Holick, M. (2021). Associação do status da vitamina D com a morbidade e mortalidade hospitalar em pacientes adultos hospitalizados com COVID-19. Endocrine Practice. 27 271e278. https://doi.org/10.1016/j.eprac.2021.02.013

COSTA, J. P. R. (2017). Vitamina D e o Envelhecimento. Trabalho Final (Mestrado) - Faculdade de Medicina, Universidade de Coimbra, Coimbra.

Dutra, J. M., Araújo, D., Favareto, R., Plácido, G., Silva, M. (2020). Suplementação alimentar da vitamina D na prevenção contra o vírus covid-19. Research, Society and Development, v. 9, n.7, p. 1-11. https://doi.org/10.33448/rsd-v9i7.4769

Figueiredo, S., José A. (2020). Covid-19, causas fundamentais, classe social e território. Trabalho, Educação e Saúde, Rio de Janeiro, v. 18, n. 3. https://doi.org/10.1590/1981-7746-sol0028

Grant, W. B., Lahore, H., McDonnell, S. L., Baggerly, C. A., French, C. B., Aliano, J. L., \& Bhattoa, H. P. (2020). Evidence that Vitamin D Supplementation Could Reduce Risk of Influenza and COVID-19 Infections and Deaths. Nutrients, 12(4), 988. https://doi.org/10.3390/nu12040988

Hammerschmidt, K. S. A., Santana, R. S. (2020). Saúde do idoso em tempos de pandemia COVID-19. Cogitare enferm. 25: e72849. http://dx.doi.org/10.5380/ce.v25i0.72849

Im, J. H., Je, Y. S., Baek, J., Chung, M. H., Kwon, H. Y., \& Lee, J. S. (2020). Nutritional status of patients with COVID-19. International journal of infectious diseases: IJID: official publication of the International Society for Infectious Diseases, 100, 390-393. https://doi.org/10.1016/j.ijid.2020.08.018

Ling, S. F., Broad, E., Murphy, R., Pappachan, J. M., Pardesi-Newton, S., Kong, M. F., \& Jude, E. B. (2020). High-Dose Cholecalciferol Booster Therapy is Associated with a Reduced Risk of Mortality in Patients with COVID-19: A Cross-Sectional Multi-Centre Observational Study. Nutrients, 12(12), 3799. https://doi.org/10.3390/nu12123799

Macaya, F., Espejo Paeres, C., Valls, A., Fernández-Ortiz, A., González del Castillo, J., Martín-Sánchez, J., Runkle, I., \& Rubio Herrera, M. Á. (2020). Interaction between age and vitamin D deficiency in severe COVID-19 infection. Nutrición Hospitalaria. https://doi.org/10.20960/nh.03193

Maghbooli Z, Sahraian MA, Ebrahimi M, Pazoki M, Kafan S. (2020). Vitamin D sufficiency, a serum 25-hydroxyvitamin D at least 30 ng/mL reduced risk for adverse clinical outcomes in patients with COVID-19 infection. PLOS ONE 15(9): e0239799. https://doi.org/10.1371/journal.pone.0239799

MOREIRA, Vanessa. Vitamina D, a nova arma contra as infeções respiratórias agudas. Rev Port Med Geral Fam, Lisboa, v. 34, n. 1, p. 48-50, fev. 2018.

Murai, I. H., Fernandes, A. L., Sales, L. P., Pinto, A. J., Goessler, K. F., Duran, C., Silva, C., Franco, A. S., Macedo, M. B., Dalmolin, H., Baggio, J., Balbi, G., Reis, B. Z., Antonangelo, L., Caparbo, V. F., Gualano, B., \& Pereira, R. (2021). Effect of a Single High Dose of Vitamin D3 on Hospital Length of Stay in Patients With Moderate to Severe COVID-19: A Randomized Clinical Trial. JAMA, 325(11), 1053-1060. https://doi.org/10.1001/jama.2020.26848

Notz, Q., Herrmann, J., Schlesinger, T., Kranke, P., Sitter, M., Helmer, P., Stumpner, J., Roeder, D., Amrein, K., Stoppe, C., Lotz, C. \& Meybohm, P. (2021). Deficiência de vitamina D em pacientes gravemente enfermos com ARDS COVID-19. Nutrição clínica (Edimburgo, Escócia, S0261-5614 (21) 001357. Publicação online avançada. https://doi.org/10.1016/j.clnu.2021.03.001 
Research, Society and Development, v. 10, n. 15, e102101522404, 2021

(CC BY 4.0) | ISSN 2525-3409 | DOI: http://dx.doi.org/10.33448/rsd-v10i15.22404

Oliveira, E. de S., Matos, MF, Cavalcante, OSS, Silvestre, JVC, Souza, DEM de, \& Morais, ACLN de. (2020). As duas faces da vitamina D como terapia adjuvante na Covid-19. InterAmerican Journal of Medicine and Health, 3. https://doi.org/10.31005/iajmh.v3i0.95.

Pizzini A., Aichner M., Sahanic S., Böhm A., Egger A., Hoermann G., Kurz K., Widmann G., Bellmann-Weiler R., Weiss G., Tancevski I., Sonnweber T., Löffler-Ragg J. (2020). Impact of Vitamin D Deficiency on COVID -19 - Uma análise prospectiva do Registro CovILD. Nutrientes. 12 (9): 2775. https://doi.org/10.3390/nu12092775

Principais itens para relatar Revisões sistemáticas e Meta-análises: A recomendação PRISMA. (2015). Epidemiologia e Serviços de Saúde, 24(2), 335342. https://doi.org/10.5123/s1679-49742015000200017

Rastogi, A., Bhansali, A., Khare, N., Suri, V., Yaddanapudi, N., Sachdeva, N., Puri, G. D., \& Malhotra, P. (2020). Suplementação de vitamina D em alta dose de curto prazo para doença COVID-19: um estudo randomizado, controlado por placebo (estudo SHADE). Postgraduate Medical Journal , postgradmedj 2020-139065. https://doi.org/10.1136/postgradmedj-2020-139065

Ricci, A., Pagliuca, A., D'Ascanio, M., Innammorato, M., De Vitis, C., Mancini, R., Giovagnoli, S., Facchiano, F., Sposato, B., Anibaldi, P., Marcolongo, A., De Dominicis, C., Laghi, A., Muscogiuri, E., \& Sciacchitano, S. (2021). Status dos níveis circulantes de vitamina D e índices prognósticos clínicos em pacientes com COVID-19. Respiratory Research, 22 (1). https://doi.org/10.1186/s12931-021-01666-3

Seijo, M; Oliveri, B. Importancia de la vitamina d en la época de covid-19. Actualizaciones en Osteología. VOL. 16 - No 2 - 2020

Sulli, A., Gotelli, E., Casabella, A., Paolino, S., Pizzorni, C., Alessandri, E., Grosso, M., Ferone, D., Smith, V., \& Cutolo, M. (2021). Vitamin D and Lung Outcomes in Elderly COVID-19 Patients. Nutrients, 13(3), 717. https://doi.org/10.3390/nu13030717

Tan, C. W., Ho, L. P., Kalimuddin, S., Cherng, B., Teh, Y. E., Thien, S. Y., Wong, H. M., Tern, P., Chandran, M., Chay, J., Nagarajan, C., Sultana, R., Low, J., $\& \mathrm{Ng}$, H. J. (2020). Estudo de coorte para avaliar o efeito da vitamina D, magnésio e vitamina B ${ }_{12}$ em combinação na progressão para desfechos graves em pacientes idosos com coronavírus (COVID-19). Nutrition (Burbank, Condado de Los Angeles, Califórnia), 79-80, 111017. https://doi.org/10.1016/j.nut.2020.111017 